\title{
The dual of non-Abelian Lattice Gauge Theory
}

\author{
Hendryk Pfeiffer ${ }^{\mathrm{a}}$ and Robert Oeckl ${ }^{\mathrm{b}}$ \\ aDepartment of Applied Mathematics and Theoretical Physics, Cambridge CB3 0WA, UK \\ ${ }^{\mathrm{b}}$ Centre de Physique Théorique, Campus de Luminy, 13288 Marseille, France
}

Non-Abelian Lattice Gauge Theory in Euclidean space-time of dimension $d \geq 2$ whose gauge group is any compact Lie group is related to a Spin Foam Model by an exact strong-weak duality transformation. The group degrees of freedom are integrated out and replaced by combinatorial expressions involving irreducible representations and intertwiners of the gauge group. This transformation is available for the partition function, for the expectation value of observables (spin networks), and for the correlator of centre monopoles which is a ratio of partition functions in the original model and an ordinary expectation value in the dual formulation.

\section{Introduction}

Exact duality transformations in Statistical Mechanics have been known since the discovery of the self-duality of the 2-dimensional Ising model. This transformation was generalized to a wider class of Abelian lattice models including Abelian Lattice Gauge Theory (LGT) $[1,2]$. In $d=4$, this transformation for pure $U(1)$ LGT can be understood as the lattice version of electric-magnetic duality. It proved to be very powerful in the analytical [3] and numerical [4] study of magnetic monopoles and their role in confinement.

We present a generalization of this transformation to non-Abelian LGT in $d \geq 2$ with a compact Lie group $G$ as the gauge group. A similar transformation has been known for the partition function in the special case of $S U(2)$ in $d=3$ [5] for which numerical algorithms are being studied [6]. For the duality transformation, all group integrals are solved, and the group degrees of freedom are replaced by combinatorial expressions involving irreducible representations and intertwiners of the gauge group. Mathematically, this transformation can be understood as an explicit calculation exploiting Tannaka-Kreǐn duality which provides a one-to-one correspondence between compact Lie groups and suitable tensor categories which arise as the categories of finitedimensional representations of these Lie groups.

In the duality transformation, the Boltzmann weight $\exp \left(-s\left(g_{P}\right)\right)$ is character expanded, gen- eralizing the Fourier expansion of the $U(1)$ case. For the standard local actions $s\left(g_{P}\right)$, such as Wilson's action or the heat kernel action, this ensures that the duality transformation still provides the strong-weak relation as for the Abelian case. The dual expressions for expectation values of generic observables can thus be used to generate their strong coupling expansion.

The dual model is a Spin Foam Model very similar to those models which are currently studied in the context of non-perturbative quantum gravity $[7,8]$ and to the state sums used in order to define topological field theory and invariants $[9,10]$. The duality transformation has been described in detail in [11] for hypercubic lattices and Lie groups. For an extension to generic triangulations and to quantum groups, see $[12,13]$.

As for the Abelian special case, we find that expectation values are mapped under the transformation to ratios of partition functions and vice versa. In particular, there is an observable of the dual model whose expectation value agrees with the correlator of centre monopoles and vortices.

\section{The duality transformation}

\subsection{The partition function}

The partition function of pure non-Abelian LGT on a $d$-dimensional hypercubic lattice reads

$$
Z=\left(\prod_{i, \mu} \int_{G} d g_{i \mu}\right) \prod_{i, \mu<\nu} f\left(g_{i \mu} g_{i+\hat{\mu} \nu} g_{i+\hat{\nu} \mu}^{-1} g_{i \nu}^{-1}\right) .
$$


Here the gauge group $G$ is any compact Lie group, $i$ denotes the lattice points and $i+\hat{\mu}$ the nearest neighbour of $i$ in the $\mu$-direction. The duality transformation for the partition function involves the expansion of the Boltzmann weight $f(g)=$ $\exp (-s(g))$ into characters,

$f(g)=\sum_{\rho} \hat{f}_{\rho} \chi^{(\rho)}(g)$.

Here the action $s(g)$ is a suitable class function of $G$. Solving all group integrals, one obtains the dual form of the partition function,

$$
\begin{aligned}
Z & =\left(\prod_{i, \mu<\nu} \sum_{\rho_{i \mu \nu}}\right)\left(\prod_{i, \mu} \sum_{P^{(i \mu)} \in \mathcal{P}_{i \mu}}\right) \\
& \times\left(\prod_{i, \mu<\nu} \hat{f}_{\rho_{i \mu \nu}}\right)\left(\prod_{i} C(i)\right) .
\end{aligned}
$$

Here the dual path integral is a sum over colourings of the plaquettes with irreducible representations $\rho_{i \mu \nu}$ and a sum over a all colourings of the links with elements of a basis $\mathcal{P}_{i \mu}$ of $G$-invariant orthogonal projectors onto the trivial component in the complete decomposition of the tensor product

$\underbrace{\left(\rho_{i-\hat{\lambda}, \lambda, \mu} \otimes \rho_{i \lambda \mu}^{*}\right) \otimes \cdots}_{\lambda \in\{1, \ldots, \mu-1\}} \otimes \underbrace{\left(\rho_{i-\hat{\nu}, \mu, \nu}^{*} \otimes \rho_{i \mu \nu}\right) \otimes \cdots}_{\nu \in\{\mu+1, \ldots, d\}}$.

The weights of the dual partition function are the dual Boltzmann weight $\hat{f}_{\rho_{i \mu \nu}}$,

$\hat{f}_{\rho}=d_{\rho} \int_{G} \overline{\chi^{(\rho)}(g)} f(g) d g$,

for each plaquette, and a group invariant

$$
\begin{gathered}
C(i)= \\
\left(\prod_{\mu<\nu} \sum_{p_{i-\hat{\mu}-\hat{\nu}, \mu, \nu}=1}^{d_{\rho_{i}-\hat{\mu}-\hat{\nu}, \mu, \nu}} \sum_{q_{i-\hat{\nu}, \mu, \nu}=1}^{d_{\rho_{i-\hat{\nu}, \mu, \nu}}} \sum_{m_{i-\hat{\mu}, \mu, \nu}=1}^{d_{\rho_{i-\hat{\mu}, \mu, \nu}}} \sum_{n_{i \mu \nu}=1}^{d_{\rho_{i \mu \nu}}}\right) \\
\left.\times \prod_{\mu=1}^{d} P_{(\underbrace{(i \mu)}_{\lambda \in\{1, \ldots, \mu-1\}}}^{\left(m_{i-\hat{\lambda}, \lambda, \mu} n_{i \lambda \mu} \ldots\right)}\right)(\underbrace{q_{i-\hat{\nu}, \mu, \nu} n_{i \mu \nu} \ldots}_{\nu \in\{\mu+1, \ldots, d\}}) \\
P_{(\underbrace{(i-\hat{\mu}, \mu)}_{\lambda \in\{1, \ldots, \mu-1\}}}^{\underbrace{\left.q_{i-\hat{\mu}, \lambda, \mu} \ldots\right)}_{i-\hat{\mu}-\hat{\lambda}, \lambda, \mu}}(\underbrace{\left.p_{i-\hat{\mu}-\hat{\nu}, \mu, \nu} m_{i-\hat{\mu}, \mu, \nu} \ldots\right)}_{\nu \in\{\mu+1, \ldots, d\}},
\end{gathered}
$$

for each lattice point. Here $d_{\rho}$ denotes the dimension of the representation $\rho$.

\subsection{Gauge invariant observables}

The generic gauge invariant observable of LGT is the spin network

$$
\begin{gathered}
W\left(\left\{\tau_{j \kappa}\right\},\left\{Q^{(j)}\right\}\right)= \\
\left(\prod_{j, \kappa} \sum_{a_{j \kappa}, b_{j \kappa}}\right)\left(\prod_{j, \kappa} t_{a_{j \kappa} b_{j \kappa}}^{\left(\tau_{j \kappa}\right)}\left(g_{j \kappa}\right)\right) \\
\times \quad\left(\prod_{j} Q_{\left(b_{j-\hat{1}, 1} \ldots b_{j-\hat{d}, d}\right),\left(a_{j 1} \ldots a_{j d}\right)}^{(j)}\right) .
\end{gathered}
$$

Here $\tau_{j \kappa}$ denotes a colouring of links with irreducible representations and

$Q^{(j)}: \bigotimes_{\mu=1}^{d} \tau_{j-\hat{\mu}, \mu} \rightarrow \bigotimes_{\mu=1}^{d} \tau_{j \mu}$,

a colouring of the lattice points with compatible intertwiners. The matrix elements of the representation $\rho$ are denoted by $t_{a b}^{(\rho)}(g)$.

The dual expression for the expectation value $\left\langle W\left(\left\{\tau_{j \kappa}\right\},\left\{Q^{(j)}\right\}\right)\right\rangle$ is given by the same expression as (3) except that the sum is over projectors in the decomposition of

$$
\begin{aligned}
& \underbrace{\left(\rho_{i-\hat{\lambda}, \lambda, \mu} \otimes \rho_{i \lambda \mu}^{*}\right) \otimes \cdots}_{\lambda \in\{1, \ldots, \mu-1\}} \\
& \otimes \underbrace{\left(\rho_{i-\hat{\nu}, \mu, \nu}^{*} \otimes \rho_{i \mu \nu}\right) \otimes \cdots}_{\nu \in\{\mu+1, \ldots, d\}} \otimes \tau_{i \mu},
\end{aligned}
$$

and the weights per point are given by

$$
\begin{aligned}
& D(i)= \\
& \left(\prod_{\mu} \sum_{a_{i \mu}=1}^{d_{\tau_{i \mu}}} \sum_{b_{i-\hat{\mu}, \mu}=1}^{d_{\tau_{i-\hat{\mu}, \mu}}}\right) Q_{\left(b_{i-\hat{1}, 1} \ldots b_{i-\hat{d}, d}\right),\left(a_{i 1} \ldots a_{i d}\right)}^{(i)} \\
& \left(\prod_{\mu<\nu} \sum_{p_{i-\hat{\mu}-\hat{\nu}, \mu, \nu}=1}^{d_{\rho_{i-\hat{\mu}}-\hat{\nu}, \mu, \nu}} \sum_{q_{i-\hat{\nu}, \mu, \nu}=1}^{d_{\rho_{i-\hat{\nu}, \mu, \nu}}} \sum_{m_{i-\hat{\mu}, \mu, \nu}=1}^{d_{\rho_{i-\hat{\mu}, \mu, \nu}}} \sum_{n_{i \mu \nu}=1}^{d_{\rho_{i \mu \nu}}}\right) \\
& \times \prod_{\mu=1}^{d} P_{(\underbrace{(i \mu)}_{\lambda \in\{1, \ldots, \mu-1\}}}^{\left(m_{i-\hat{\lambda}, \lambda, \mu} n_{i \lambda \mu} \ldots\right)}(\underbrace{q_{i-\hat{\nu}, \mu, \nu} n_{i \mu \nu} \ldots}_{\nu \in\{\mu+1, \ldots, d\}}) a_{i \mu} \\
& P_{(\underbrace{(i-\hat{\mu}, \mu)}_{\lambda \in\{1, \ldots, \mu-1\}}}^{\underbrace{p_{i-\hat{\mu}-\hat{\lambda}, \lambda, \mu} q_{i-\hat{\mu}, \lambda, \mu}})}(\underbrace{\left.p_{i-\hat{\mu}-\hat{\nu}, \mu, \nu} m_{i-\hat{\mu}, \mu, \nu} \ldots\right)}_{\nu \in\{\mu+1, \ldots, d\}}) b_{i-\hat{\mu}, \mu} .
\end{aligned}
$$


The normalized expectation value $\left\langle W\left(\left\{\tau_{j \kappa}\right\},\left\{Q^{(j)}\right\}\right)\right\rangle$ of the spin network is therefore mapped to a ratio of partition functions in the dual model.

\subsection{Monopole and vortex correlators}

A similar transformation is available for the correlator of centre monopoles and vortices. It is given by a ratio of partition functions $Z(X) / Z$,

$$
\begin{aligned}
Z(X) & =\left(\prod_{i, \mu} \int_{G} d g_{i \mu}\right) \\
& \times \prod_{i, \mu<\nu} f\left(g_{i \mu} g_{i+\hat{\mu} \nu} g_{i+\hat{\nu} \mu}^{-1} g_{i \nu}^{-1} X_{i \mu \nu}\right),
\end{aligned}
$$

where $X_{i \mu \nu}$ associates an element of the centre $Z(G)$ to each plaquette. Its dual expression has the form of an expectation value under the dual path integral (3),

$\left.Z(X)=\left\langle\prod_{i, \mu<\nu} \tilde{t}^{\left(\rho_{i \mu \nu}\right)}\left(X_{i \mu \nu}\right)\right)\right\rangle_{\text {dual }}$

where $\tilde{t}^{(\rho)}$ denotes the matrix element of the representation of $Z(G)$ that is induced by $\rho$.

\section{Some comments}

It should be pointed out that the dual Boltzmann weight $\hat{f}_{\rho}$ is strictly positive for the standard actions. However, the coefficients $C(i)$ and $D(i)$ are not, so that their sign has to be associated to the observable while their modulus can be part of the probability density.

The heat kernel action is defined by its dual Boltzmann weight $\hat{f}_{\rho}=d_{\rho} \cdot \exp \left(-C_{\rho} / \beta\right)$ where $C_{\rho}$ is the eigenvalue of the second order Casimir operator in the representation $\rho$. The configurations of the dual path integral can therefore be ordered according to their $\beta$-dependence. One just sorts them by the value of the sum of the second order Casimir eigenvalues of the representations that are attached to the plaquettes. The strong coupling expansion of the generic spin network expectation value can thus be read off from its dual expression.

Interesting future applications of the duality transformation include not only the study of the topological degrees of freedom in connection with an understanding of confinement, but also the development of efficient algorithms for the dual model. Finally, the relation of the dual form of LGT with models studied in the context of nonperturbative quantum gravity suggests that both areas can benefit from a combination of the available techniques, numerically and analytically.

\section{Acknowledgements}

R.O. is grateful to NATO for a Research Fellowship, H.P. would like to thank Emmanuel College, Cambridge, for a Research Fellowship. Thanks are also due to I. Drummond, J. Jersák, A. J. Macfarlane, S. Majid and T. Neuhaus for valuable discussions.

\section{REFERENCES}

1. R. Savit, Rev. Mod. Phys. 52, No. 2 (1980) 453-487.

2. M. E. Peskin, Ann. Phys. 113 (1978) 122152.

3. J. Fröhlich and P.-A. Marchetti, Europhys. Lett. 2, No. 12 (1986) 933-940.

4. J. Jersák, T. Neuhaus and H. Pfeiffer, Phys. Rev. D 60, No. 5 (1999) 054502, heplat/9903034.

5. R. Anishetty, S. Cheluvaraja, H. S. Sharatchandra and M. Mathur, Phys. Lett. B 314 (1993) 387-390, hep-lat/9210024.

6. N. D. Hari Dass and D.-S. Shin, Nucl. Phys. Proc. Suppl. B 94 (2001) 670-674, heplat/0011038.

7. J. C. Baez, in Lecture Notes in Physics 543, gr-qc/9905087.

8. D. Oriti, gr-qc/0106091.

9. V. G. Turaev and O. Y. Viro, Topology 31, No. 4 (1992) 865-902.

10. L. Crane, L. H. Kauffmann and D. N. Yetter, J. Knot Th. Ramif. 6, No. 2 (1997) 177-234, hep-th/9409167.

11. R. Oeckl and H. Pfeiffer, Nucl. Phys. B 598, No. 1-2 (2001) 400-426, hep-th/0008095.

12. R. Oeckl, Generalized lattice gauge theory, spin foams and state sum invariants, in preparation.

13. H. Pfeiffer, J. Math. Phys., in press, hepth/0106029. 